\title{
Estudio comparativo del maíz morado (Zea mays $L$.) y simvastatina en la reducción de lípidos séricos de pacientes diabéticos normotensos con dislipidemia
}

\author{
Comparative study of Peruvian purple corn (Zea mays L.) and simvastatin in serum lipids \\ reduction in normotense dyslipidemic diabetics
}

\begin{abstract}
Gerardo Ronceros ${ }^{1,2}$, Willy Ramos ${ }^{1}$, Jorge Arroyo ${ }^{1}$, Carlos Galarza ${ }^{1}$, Ericson L. Gutiérrez¹, Alex G. Ortega-Loayza ${ }^{3}$, Christian La Rosa², Carolina Cucho², Luis Palma²

${ }^{1}$ Instituto de Investigaciones Clínicas. Facultad de Medicina, Universidad Nacional Mayor de San Marcos. Lima - Perú.

2 Departamento de Patología Clínica. Hospital Nacional Dos de Mayo. Lima - Perú.

${ }^{3}$ Department of Internal Medicine. Virginia Commonwealth University, Richmond, VA, USA.
\end{abstract}

\section{Resumen}

Objetivos: Determinar la eficacia de la administración del maíz morado (Zea mays) en la reducción de los niveles de lípidos séricos (efecto hipolipemiante) en pacientes diabéticos no hipertensos con dislipidemia. Comparar el efecto hipolipemiante del maíz morado con simvastatina. Diseño: Ensayo clínico. Institución: Instituto de Investigaciones Clínicas, Facultad de Medicina, Universidad Nacional Mayor de San Marcos, Lima, Perú. Participantes: Pacientes diabéticos dislipidémicos no hipertensos. Intervenciones: Entre enero y octubre 2006, se formó aleatoriamente dos grupos de investigación de 15 pacientes diabéticos dislipidémicos no hipertensos, de diagnóstico reciente, sin tratamiento previo y sin complicaciones crónicas; un grupo recibió simvastatina $40 \mathrm{mg} / \mathrm{dia}$ y el otro grupo maíz morado (comprimidos deshidratados y micropulverizados $1 \mathrm{~g} / \mathrm{dia}$ ), por un período de 30 días. Los pacientes paralelamente realizaron dieta hipograsa con evaluación nutricional semanal y recibieron el tratamiento hipoglicemiante convencional con glibenclamida. Se realizo dosaje basal y postratamiento de lípidos séricos, glucosa basal, hemoglobina glicosilada, pruebas de función hepática y renal. E análisis estadístico se efectuó con la prueba t de student, con un intervalo de confianza de 95\%. Principales medidas de resultados: Modificación de los valores séricos de colesterol, triglicéridos y glucosa. Resultados: Se observó que en ambos grupos se produjo reducción en los valores séricos de colesterol total, colesterol LDL, triglicéridos y glucosa en ayunas; asimismo, aumento de los niveles séricos de colesterol HDL. Al compararse los resultados basales y postratamiento para maiz morado, se encontró reducción significativa de los valores de triglicéridos y aumento significativo del colesterol HDL. Para el grupo tratado con simvastatina, hubo reducción estadísticamente significativa del colesterol total, colesterol LDL, triglicéridos y aumento estadísticamente significativo del colesterol HDL, en comparación con los valores basales. El maíz morado mostró optimizar el control de la glucosa, efecto muy superior y estadísticamente significativo en comparación con la simvastatina. Se encontró diferencia estadísticamente significativa para los valores postratamiento de colesterol total, colesterol LDL, colesterol HDL y triglicéridos a favor del grupo que recibió simvastatina en comparación con el que recibió maíz morado. Conclusiones: A la dosis estudiada, el maíz morado mostró ser eficaz para reducir los niveles de triglicéridos, aumentar el colesterol HDL y optimizar el control de la glucosa en pacientes diabéticos no hipertensos. Simvastatina fue más eficaz que el maiz morado en el tratamiento de la dislipidemia, sin efectos importantes sobre la glicemia.

Palabras clave: Diabetes mellitus, Zea mays, simvastatina, dislipidemias.

Abstract

Objectives: To determine the effectiveness of Peruvian purple corn (Zea mays) administration in serum lipids levels reduction (hypolipidemic effect) in non hypertensive diabetic patients with dyslipidemia; to compare hypolipidemic effect of Peruvian purple corn and simvastatin. Design: Clinical essay. Setting: Instituto de Investigaciones Clinicas, Facultad de Medicina, Universidad Nacional Mayor de San Marcos, Lima, Peru. Participants: Non hypertensive dyslipidemic diabetic patients. Interventions: Between January and October 2006 two groups of 15 non hypertensive dyslipidemic diabetic patients were randomly formed, patients with recent diagnosis, without previous treatment and without chronic complications; one group recieved simvastatin $40 \mathrm{mg} / \mathrm{day}$ and the other group Peruvian purple corn (dehydrated and micropulverized pills $1 \mathrm{~g} /$ day) for 30 days. Patients had low fat diet with weekly nutritional evaluation and received conventional hypoglicemic treatment with glibenclamide. Serum lipids, glucose, glycosylated hemoglobin, hepatic and renal function tests were done before and after treatment. Statistical analysis used Student $t$ test with $95 \%$ confidence interval. Main outcome measures: Serum cholesterol, triglycerids and glucose levels variation. Results: There was reduction in both groups of total cholesterol, LDL cholesterol, triglycerides and fasting glucose serum levels; HDL cholesterol serum levels increased. Comparing basal and post treatment results for purple corn, there was significant reduction of triglycerides levels and significant increase of HDL cholesterol. In the simvastatin group there was significant statistical reduction of total cholesterol, LDL cholesterol, triglycerides and statistically significant increase in HDL cholesterol compared with basal levels. Compared with simvastatin purple corn optimized glucose control more significantly. There was significant statistical difference for post treatment values of total cholesterol, LDL cholesterol, HDL cholesterol and triglycerides in favor of the group that received simvastatin compared with purple corn. Conclusions: At doses studied Peruvian purple corn was effective in reducing triglyceride levels, increasing HDL cholesterol and optimizing glucose control in non hypertensive diabetic patients. Simvastatin was more efficacious than Peruvian purple corn in treatment of dyslipidemia without important effects on glycemia.

Key words: Diabetes mellitus, Zea mays, simvastatin, dyslipidemias.

An Fac med. 2012;73(2):113-7 


\section{INTRODUCCIÓN}

La diabetes mellitus es un síndrome crónico no curable con los medios disponibles en la actualidad. Se caracteriza por una pérdida de la homeostasis de la glucosa, lo cual también implica trastornos en el metabolismo de los lípidos y las proteínas. Sus síntomas causan limitaciones en el modo de vida de los pacientes y, en muchos de ellos, el desarrollo de complicaciones crónicas que pueden llevarlos a la invalidez o a la muerte ${ }^{(1-6)}$.

El estrés oxidativo es un factor importante para el desarrollo y progresión de la enfermedad coronaria en este grupo de pacientes ${ }^{(7-9)}$. La oxidación de las lipoproteínas de densidad baja (LDL) es un paso temprano importante para el desarrollo de aterosclerosis y autoanticuerpos contra la LDL oxidada. Los pacientes diabéticos tienden a tener una proporción alta de radicales libres, lo cual los hace más susceptibles a la oxidación de la LDL y al riesgo de eventos cardiovasculares ( 2 a 7 veces más alto que en personas no diabéticas) ${ }^{(9-18)}$.

Estudios recientes han mostrado que alimentos ricos en antocianinas tienen actividad antioxidante y mejoran los perfiles lipídicos en modelos experimentales de hiperlipidemia ${ }^{(19,20)}$.

El maíz morado (Zea mays L.) es una variedad de maíz que es originaria del Perú y Bolivia. Diversas investigaciones revelan que contiene un número importante de grupos fenólicos y flavonoides llamados antocianinas. De este modo, las antocianinas son usadas como colorantes naturales que son atóxicos, no teratogénicos y no mutagénicos ${ }^{(21,22)}$. Los compuestos fenólicos y las antocianinas son antioxidantes que protegen las membranas celulares y el ADN de los efectos de los radicales libres.

Hagiwara y col. (2001) demostraron los efectos antineoplásicos de las antocianinas del maíz morado en animales de experimentación, encontrando que previene la carcinogénesis por 2-amino-1-metil-6-fenilimidazo [4,5-b] piridina (radical libre que pertenece al grupo de las nitrosaminas). El resultado fue que de las 20 ratas a las que se les administró solamente la sustancia cancerígena, 17 (el 85\%) desarrollaron cáncer de intestino grueso frente a solo 8 ratas (40\%) que recibieron maíz morado (antocianinas 5\%) ${ }^{(23)}$.

Tsuda y col. (2003) demostraron que la dieta con maíz morado rico en antocianinas puede tener beneficio en la prevención de la obesidad y diabetes en ratones, ya que las alteraciones inducidas por una dieta alta en grasas (hiperglicemia, hiperinsulinemia e hiperleptinemia) fue normalizada en el grupo que recibió adicionalmente maíz morado. Además, la dieta rica en maíz morado suprimió los niveles de ARN mensajero comprometidos en la síntesis de ácidos grasos y triglicéridos ${ }^{(24)}$.

Arroyo y col. (2005) demostraron en dos estudios las propiedades hipocolesterolémica y antioxidante del extracto hidroalcohólico atomizado de maíz morado (Zea mays L.) a dosis de 250, 500 y $1000 \mathrm{mg} / \mathrm{kg}$, en ratas Holtzmann, a las que se indujo experimentalmente hipercolesterolemia ${ }^{(25,26)}$.

De este modo, el maíz morado tendría propiedades antioxidantes e hipolipemiantes y anticancerígenas. Es por esta razón que se plantea evaluar los beneficios hipolipemiantes del maíz morado en pacientes diabéticos, dislipidémicos no hipertensos.

\section{MÉTODOS}

El presente estudio experimental, prospectivo, comparativo fue realizado en el Instituto de Investigaciones Clínicas de la Universidad Nacional Mayor de San Marcos, entre enero y octubre del año 2006.

Se incluyó en el estudio a pacientes diabéticos con dislipidemia mixta, de diagnóstico reciente, sin tratamiento previo, con dislipidemia, no hipertensos, de edad entre 40 y 60 años e índice de masa corporal de 25 a 40. Se excluyó a los pacientes que se encontraban usando $\beta$-bloqueadores o diuréticos y con enfermedad crónica asociada a diabetes mellitus (enfermedad cardiovascular, renal, neuropatía), pacientes que realizaban ejercicio con una frecuencia mayor o igual a 5 veces por semana, que hubieran recibido tratamiento previo para dislipidemia, mujeres que se encontraran gestando o durante el período de lactancia.

La muestra estuvo constituida por 30 pacientes diabéticos no hipertensos con dislipidemia. Luego de la captación y estandarización, los pacientes fueron distribuidos aleatoriamente a uno de los siguientes grupos de investigación: a) grupo A, constituido por 15 diabéticos dislipidémicos no hipertensos que recibieron comprimidos de Zea mays; y grupo $\mathrm{B}$, constituido por 15 diabéticos dislipidémicos no hipertensos que recibieron tratamiento con simvastatina.

Antes de iniciar el estudio, se explicó a los pacientes y tuvieron la oportunidad de realizar preguntas y discutir acerca del estudio. Luego de ello, se procedió a la firma del consentimiento informado. Se obtuvo muestras de sangre en ayunas para el dosaje de lípidos séricos (colesterol total, colesterol LDL, colesterol VLDL, colesterol HDL y triglicéridos), glucosa basal, hemoglobina glicosilada, pruebas de función hepática y renal.

Se indicó una dieta para diabético, la cual fue realizada por los pacientes durante el transcurso del estudio, de contenido bajo en lípidos y evaluación nutricional semanal. Luego, se procedió a la administración de comprimidos de simvastatina (40 mg/día) para el grupo B y maíz morado (Zea mays) deshidratado y micropulverizado en cápsulas de $1 \mathrm{~g}$ ( $\mathrm{g} /$ día $)$ para el grupo A, por un período de 30 días, luego de los cuales se realizó una evaluación final en la que se obtuvo muestras de sangre con la finalidad de determinar si existía variación significativa de los niveles de lípidos séricos, glucosa basal, pruebas de función hepática y renal. Por último, se determinó y comparó la eficacia y seguridad de ambos tratamientos. 
Se realizó estadística univariada, obteniéndose frecuencias, porcentajes, medidas de tendencia central y de dispersión. La estadística bivariada se realizó con la prueba t de Student, haciendo los cálculos con un intervalo de confianza del $95 \%$.

\section{RESULTADOS}

La determinación basal de los niveles séricos de colesterol total, colesterol HDL, triglicéridos y glucosa en ayunas en los grupos de pacientes asignados a recibir tratamiento con maíz morado (grupo A) y simvastatina (grupo B) no mostraron diferencias estadísticamente significativas, lo cual puede observarse en la tabla 1.

$\mathrm{Al}$ evaluarse la respuesta a los tratamientos administrados se halló que en ambos grupos de investigación se produjo una reducción en los valores séricos de colesterol total, colesterol LDL, triglicéridos y glucosa en ayunas; asimismo, en ambos grupos se observó también un aumento de los niveles séricos de colesterol HDL.

Al compararse los resultados basales y postratamiento para maíz morado, se encontró diferencia estadísticamente significativa para la reducción de los valores de triglicéridos y en el aumento significativo del colesterol HDL; existió también una reducción no significativa ( $p>0,05)$ de los niveles séricos de colesterol total y colesterol LDL. Para el grupo tratado con simvastatina, se produjo una reducción estadísticamente significativa del colesterol total, colesterol LDL, triglicéridos y un aumento estadísticamente significativo del colesterol HDL, en comparación con los valores basales (tabla 3).

Se encontró diferencia estadísticamente significativa para los valores post tratamiento de colesterol total, colesterol LDL y colesterol HDL a favor del grupo que recibió simvastatina en comparación con el que recibió maíz morado; los demás parámetros evaluados no mostraron diferencia
Tabla 1. Medición basal (en $\mathrm{mg} / \mathrm{dL}$ ) de colesterol total, LDL, HDL y glucosa en ayunas de pacientes de ambos grupos de investigación.

\begin{tabular}{cccc} 
Medición basal & Maíz morado & Simvastatina & Valor $p$ \\
Colesterol total & $320,6 \pm 72,0$ & $310,9 \pm 44,4$ & 0,661 \\
Colesterol HDL & $35,6 \pm 6,8$ & $35,4 \pm 6,2$ & 0,933 \\
Colesterol LDL & $166,3 \pm 27,7$ & $181,1 \pm 23,1$ & 0,125 \\
Triglicéridos & $342,4 \pm 101,7$ & $358,8 \pm 78,5$ & 0,625 \\
Glucosa en ayunas & $211,3 \pm 61,9$ & $177,8 \pm 41,5$ & 0,092 \\
\hline
\end{tabular}

Tabla 2. Medición postratamiento (mg/dL) del colesterol total, LDL, HDL y glucosa en ayunas de pacientes de ambos grupos de investigación.

\begin{tabular}{cccc} 
Post tratamiento & Maíz morado & Simvastatina & Valor $p$ \\
Colesterol total & $291,3 \pm 52,8$ & $201,9 \pm 26,7$ & $<0,001$ \\
Colesterol HDL & $38,4 \pm 5,5$ & $38,8 \pm 7,8$ & 0,872 \\
Colesterol LDL & $156,3 \pm 24,8$ & $130,3 \pm 17,8$ & 0,003 \\
Triglicéridos & $324,4 \pm 94,2$ & $276,4 \pm 50,7$ & 0,093 \\
Glucosa en ayunas & $112,5 \pm 15,2$ & $116,5 \pm 20,0$ & 0,542 \\
\hline
\end{tabular}

Tabla 3. Niveles de lípidos séricos antes y después del tratamiento con maíz morado y simvastatina.

\begin{tabular}{|cccc} 
& Pretratamiento & Postratamiento & Valor P \\
Grupo maíz morado & & & \\
\hline Colesterol total & $320,6 \pm 72,0$ & $291,3 \pm 52,8$ & 0,055 \\
Colesterol HDL & $35,6 \pm 6,8$ & $38,4 \pm 5,5$ & 0,030 \\
Colesterol LDL & $166,3 \pm 27,7$ & $156,3 \pm 24,8$ & 0,066 \\
Triglicéridos & $342,4 \pm 101,7$ & $324,4 \pm 94,2$ & 0,026 \\
\hline Grupo simvastatina & & & \\
\hline Colesterol total & $310,9 \pm 44,4$ & $201,9 \pm 26,7$ & $<0,001$ \\
Colesterol HDL & $35,4 \pm 6,2$ & $38,8 \pm 7,8$ & 0,035 \\
Colesterol LDL & $181,1 \pm 23,1$ & $130,3 \pm 17,8$ & $<0,001$ \\
Triglicéridos & $358,8 \pm 78,5$ & $276,4 \pm 50,7$ & 0,001 \\
\hline
\end{tabular}

Tabla 4. Comparación (\%) del efecto del maíz morado y simvastatina postratamiento y basal.

\begin{tabular}{ccc} 
Post tratamiento & Maíz morado & Simvastatina \\
Colesterol total & $\downarrow 9,0 \%$ & $\downarrow 35,1 \%$ \\
Colesterol HDL & $\uparrow 7,9 \%$ & $\uparrow 9,6 \%$ \\
Colesterol LDL & $\downarrow 6,1 \%$ & $\downarrow 30,5 \%$ \\
Triglicéridos & $\downarrow 5,3 \%$ & $\downarrow 22,9 \%$ \\
Glucosa en ayunas & $\downarrow 46,8 \%$ & $\downarrow 34,5 \%$ \\
\hline
\end{tabular}


significativa (tabla 2). La mayor reducción la obtuvo la simvastatina para el colesterol total $(35,1 \%)$ y colesterol LDL $(30,5 \%)$, mientras que en el grupo que recibió maíz morado se observó un mejor control de la glicemia en ayunas, obteniéndose una reducción de 46,8\% de los niveles basales (tabla 4).

Ambos tratamientos fueron bien tolerados por los pacientes, observándose efectos adversos en $13,3 \%$ de pacientes que recibieron maíz morado (estreñimiento) y en $20 \%$ de los que recibieron simvastatina (dolor abdominal, mareos y náuseas); no se presentaron reacciones adversas graves en ninguno de los grupos de investigación. Al final del tratamiento, los niveles séricos de transaminasa glutámico pirúvica (TGP) y creatinina no sufrieron variaciones significativas, manteniéndose en rango normal en ambos grupos (tabla 5).

\section{DISCUSIÓN}

Se ha realizado diversas investigaciones en animales de experimentación que muestran que el maíz morado tiene actividad hipolipemiante. El presente trabajo constituye la primera investigación clínica que demuestra la eficacia del maíz morado en la reducción significativa de los niveles séricos de triglicéridos y en el aumento de los niveles de colesterol HDL; para el caso del colesterol total y colesterol LDL se observó una reducción no significativa ( $9,0 \%$ y $6,1 \%$, respectivamente); con respecto al valor basal, simvastatina mostró reducción significativa tanto de los valores de colesterol total, colesterol LDL y triglicéridos con un aumento significativo de los niveles de colesterol HDL.

Al compararse la eficacia de ambos fármacos, se observó que la simvastatina fue más eficaz que el maíz morado en la reducción del colesterol total, colesterol LDL, triglicéridos y en el aumento del colesterol LDL. Tomando en cuenta que ambos grupos recibieron tratamiento hipoglicemiante oral con el mismo fármaco (glibenclamida) y fueron sometidos a dieta, el maíz morado mostró optimizar el control de la glucosa, efecto que fue muy superior y estadísticamente significativo en comparación con la simvastatina.

Los resultados de este estudio difieren parcialmente con los obtenidos en estudios preclínicos realizados por Arroyo y col. en ratas Holtzmann. En un primer estudio, Arroyo encontró una reducción no significativa de los niveles de colesterol $(21,5 \%)$ y aumento de colesterol HDL (27\%) en animales de experimentación, con reducción significativa de los niveles de radicales libres ${ }^{(25)}$; mientras que, en un segundo estudio, el maíz morado indujo una reducción significativa de los niveles séricos de colesterol total y reducción no significativa de los niveles de triglicéridos. Los resultados de estos dos últimos estudios pueden haberse visto limitados por la pequeña muestra incluida en cada grupo de investigación $(n=6)^{(26)}$.

Se espera además que el maíz morado tenga efectos importantes en la prevención de la enfermedad coronaria, no solo por la mejoría de los valores de lípidos séricos sino también por su

Tabla 5. Mediciones basal y postratamiento (en $\mathrm{mg} / \mathrm{dL}$ ) de colesterol total, LDL, HDL y glucosa en ayunas de pacientes de ambos grupos de investigación.

\begin{tabular}{cccc} 
Medición basal & Maíz morado & Simvastatina & Valor $p$ \\
\hline TGP basal & $33,7 \pm 11,3$ & $32,9 \pm 10,5$ & 0,829 \\
TGP post tratamiento & $30,0 \pm 10,7$ & $28,7 \pm 9,7$ & 0,723 \\
Creatinina basal & $1,1 \pm 0,3$ & $0,9 \pm 0,2$ & 0,179 \\
Creatinina postratamiento & $0,9 \pm 0,2$ & $0,9 \pm 0,2$ & 0,103 \\
\hline
\end{tabular}

TGP = transaminasa glutámico pirúvica. efecto antioxidante, que podría ser importante en la disminución de la oxidación de las LDL, paso importante en la aterogénesis ${ }^{(10-13)}$; la demostración de esto requiere la realización de ensayos clínicos controlados, prospectivos, de larga duración y seguimiento.

El maíz morado ha mostrado además ser una alternativa segura, puesto que no se observó efectos adversos graves en los pacientes tratados y desde el punto de vista bioquímico no se produjo alteración de las pruebas de función hepática ni renal, siendo similar en este punto a los resultados encontrados para la simvastatina.

En conclusión, a la dosis estudiada, el maíz morado mostró ser eficaz y seguro en la reducción de los niveles de triglicéridos, en el aumento del colesterol HDL y optimizó el control de la glucosa en pacientes diabéticos no hipertensos. Simvastatina fue más eficaz que el maíz morado en el tratamiento de la dislipidemia sin efectos importantes sobre la glicemia.

\section{REFERENCIAS BIBLIOGRÁFICAS}

1. World Health Organization: Definition, Diagnosis, and Classification of Diabetes Mellitus and Its Complications: Report of WHO Consultation. Part 1. Diagnosis and Classification of Diabetes Mellitus. World Health Org., Geneva, 1999.

2. Warren RE. The stepwise approach to the management of type 2 diabetes. Diabetes Res Clin Pract. 2004;65S:S3-S8.

3. American Diabetes Association: Evidence-based nutrition principles and recommendations for the treatment and prevention of diabetes and related complications. Diabetes Care. 2003;26 (Suppl 1):S51-S61.

4. Zimmet P, Alberti KG, Shaw J. Global and societal implications of the diabetes epidemic. Nature. 2001;414:782-7.

5. American Diabetes Association. Standards of medical care in diabetes. Diabetes Care. 2005;28 Suppl 1:S4-36.

6. King $\mathrm{H}$, Aubert RE, Herman WH. Global burden of diabetes, 1995 - 2025. Diabetes care. 1998;21(9):1414-31.

7. Uzel N, Sivas A, Uysal M. Erytrocyte lipid peroxidation and glutathione peroxidase activities in patients with diabetes mellitus. Horm Metab Res. 1987;19:89-90.

8. Mamposo M, León O, Licea M, Pérez B, Castillo $R$. Especies reactivas de oxígeno en la diabetes mellitus con retinopatía y sin ella. Rev Cubana End. 1999;10:8-15

9. Li LF, Li J. Link between oxidative stress and insulin resistance. Chin Med Sci J. 2007;22:254-9. 
10. Kreisberg RA, Oberman A. Medical management of hyperlipidemia/dyslipidemia. J Clin Endocrinol Metab. 2003;88:2445-61.

11. American Diabetes Association. Dyslipidemia management in adults with diabetes. Diabetes Care. 2004;27:s68-s71.

12. Wahle KWJ. Atherosclerosis: cell biology and lipoproteins. Curr Opin Lipidol. 2002;13:347-9.

13. Kural BV, Orem C, Uydu HA, Alver A, Orem A. The effects of lipid-lowering therapy on paraoxonase activities and their relationships with the oxidantantioxidant system in patients with dyslipidemia. Coron Artery Dis. 2004;15:277-83.

14. Klein R. Kelly West Lecture 1994: hyperglycemia and microvascular and macrovascular disease in diabetes. Diabetes Care. 1995;18:258-68.

15. Kleinman JC, Donahue RP, Harris MI, Finucane FF, Madans JH, Brock DB. Mortality among diabetics in a national sample. Am J Epidemiol. 1988;128:389-401.

16. Butler WJ, Ostrander LD, Carman WJ, Lamphiear DE. Mortality from coronary heart disease in the Tecumseh Study: Iong-term effect of diabetes mellitus, glucose tolerance and other risk factors. Am J Epidemiol. 1985;121:541-7.

17. Howard BV. Macrovascular complications of diabetes mellitus. En: LeRoith D, Taylor SI, Olefsky,
JM eds. Diabetes Mellitus: A Fundamental and Clinical Text. Philadelphia, PA: Lippincott Williams \& Wilkins; 1996:792-7.

18. Kannel WB, McGee DL. Diabetes and glucose tolerance as risk factors for cardiovascular disease: the Framingham Study. Diabetes Care. 1979;2:120-6.

19. Xia X, Ling W, Ma J, Xia M, Hou M, Wang Q, et al. An anthocyanin-rich extract from black rice enhances atherosclerotic plaque stabilization in apolipoprotein Edeficient mice. J Nutr. 2006;136:2220-5.

20. Aviram M, Rosenblat M, Gaitini D, Nitecki S, Hoffman $A$, Dornfeld L, et al. Pomegranate juice consumption for 3 years by patients with carotid artery stenosis reduces common carotid intima-media thickness, blood pressure and LDL oxidation. Clin Nutr. 2004;23:423-33.

21. Shimizu T, Nakamura M. Anthocyanins. En: M Fuji (Ed.). Gaisetsu Shokuyou Tennennshikiso, Korin, Tokyo, 1993:71-104.

22. Sano M, Tamano S, Hagiwara A, kagawe M Nakamura M, Imaida K, Hirose M. 13-week oral toxicity study of pigments extracted form the purple sweet potato in F344/DuCrj rats. Jpn J Food Chem 1996;3:99-105.

23. Pronounced inhibition by a natural anthocyanin, purple corn color, of 2-amino-1-methyl-6-phenylimidazo $[4,5-b]$ pyridine (PhIP)- associated colorectal car- cinogenesis in male F344 rats pretreated with 1,2dimethylhydrazine. Cancer Lett. 2001;171:17-25.

24. Tsuda T, Horio F, Uchida K, Aoki H, Osawa T. Dietary cyanidin 3-O- $\beta$-D-glucoside-rich purple corn color prevents obesity and ameliorates hyperglycemia in mice. J Nutr. 2003:133:2125-30.

25. Arroyo J, Rojas J, Chumpitaz G, Burga J, De la Cruz W, Valencia J y col. Actividad hipocolesterolémica y antioxidante del extracto hidroalcohólico atomizado de Zea mays $L$ (maiz morado) en ratas inducidas con colesterol. An Fac med. 2005;66:S20.

26. Arroyo J, Ráez E, Rodríguez M, Chumpitaz V, Burga J, De la Cruz W. Reducción del colesterol y aumento de la capacidad antioxidante por el consumo crónico de maiz morado (Zea mays $L$ ) en ratas hipercolesterolémicas. Rev Med Exp Salud Publica. 2007;24:157-62.

Artículo recibido el 23 de enero de 2012 y aceptado para publicación el 8 de marzo de 2012.

Correspondencia:

Dr. Gerardo Ronceros Medrano

Correo electrónico: gerardo_ronceros@hotmail.com 